\title{
Programmed cell death in amyotrophic lateral sclerosis
}

\author{
Christelle Guégan ${ }^{1,2}$ and Serge Przedborski ${ }^{1,3,4}$ \\ ${ }^{1}$ Department of Neurology, Columbia University, New York, New York, USA \\ ${ }_{2}^{2}$ Institut National de la Santé et de la Recherche Médicale, Unit 421, Institut Mondor de Médecine Moléculaire, Créteil, France \\ ${ }^{3}$ Department of Pathology, and \\ ${ }^{4}$ Center of Neurobiology and Behavior, Columbia University, New York, New York, USA \\ J. Clin. Invest. 111:153-161 (2003). doi:10.1172/JCI200317610.
}

Amyotrophic lateral sclerosis (ALS) is a relentless fatal paralytic disorder confined to the voluntary motor system (1). Its prevalence is about three to five in 100,000 individuals, making it the most frequent paralytic disease in adults. Although ALS can strike anyone at any age, generally the onset of the disease is in the fourth or fifth decade of life. Common clinical features of ALS include muscle weakness, fasciculations, brisk (or depressed) reflexes, and extensor plantar responses. Even though motor deficit usually predominates in the limbs, bulbar enervation can also be severely affected, leading to atrophy of the tongue, dysphagia, and dysarthria. Other cranial nerves (e.g., oculomotor nerves) are usually spared. The progressive decline of muscular function results in paralysis, speech and swallowing disabilities, emotional disturbance, and, ultimately, respiratory failure causing death among the vast majority of ALS patients within 2-5 years after the onset of the disease. Pathologically, ALS is characterized by a loss of upper motor neurons in the cerebral cortex and of the lower motor neurons in the spinal cord. Often, there is also a profound degeneration of the corticospinal tracts, which is most evident at the level of the spinal cord. The few remaining motor neurons are generally atrophic, and many demonstrate abnormal accumulation of neurofilament, in both their cell bodies and axons. To date, only a few approved treatments (e.g., mechanical ventilation and riluzole) prolong survival in ALS patients to some extent. However, the development of more effective neuroprotective therapies remains impeded by our limited knowledge of the actual mechanisms by which neurons die in ALS, and of how the disease progresses and propagates.

\footnotetext{
Address correspondence to: Serge Przedborski, BB-307, Columbia University, 650 West 168th Street, New York, New York 10032, USA. Phone: (212) 305-1540; Fax: (212) 305-5450; E-mail: SP30@columbia.edu.

Conflict of interest: The authors have declared that no conflict of interest exists.

Nonstandard abbreviations used: amyotrophic lateral sclerosis (ALS); copper/zinc superoxide dismutase (SOD1); pheochromocytoma-12 (PC-12); programmed cell death (PCD); extracellular signal-regulated kinase (ERK); neurotrophin receptor (NTR); apoptotic protease-activating factor-1 (Apaf-1).
}

ALS, like other common neurodegenerative disorders, is sporadic in the vast majority of patients, and familial in only a few (1). The clinical and pathological expressions of ALS are almost indistinguishable between the familial and sporadic forms, although often in the former the age at onset is younger, the course of the disease more rapid, and the survival after diagnosis shorter (1). The cause of sporadic ALS remains unknown, while that of at least some familial forms has been identified (see below). Although the identified gene defects responsible for ALS account for a minute fraction of cases, most experts believe that unraveling the molecular basis by which those mutant gene products cause neurodegeneration may shed light on the etiopathogenesis of the common sporadic form of ALS.

\section{Genetic forms of ALS}

Although familial ALS is often referred to as a single entity, genetic evidence actually reveals at least four different types that have been assigned to distinct loci of the human genome (2). This review will focus on a form that is responsible for the disease in approximately $20 \%$ of all familial cases and that is linked to mutations in the gene for the cytosolic free radical-scavenging enzyme superoxide dismutase- 1 (SOD1) $(3,4)$. To date, approximately 100 different point mutations in SOD1 throughout the entire gene have been identified in ALS families, and all but one is dominant. Many of these mutations lead to the substitution of an amino acid within regions of the enzyme with very distinct structural and functional roles. It is thus fascinating to note that so many discrete SOD1 alterations share a similar clinical phenotype, even though the disease duration and, to a lesser extent, age at onset vary among patients with different SOD1 mutations (5). Also astonishing is the fact that SOD1 mutations, which are present at birth and, by virtue of SOD1's ubiquitous expression, in all tissues, produce a rapidly progressive adult-onset degenerative condition in which motor neurons are almost exclusively affected.

Most of these mutations have apparently reduced enzymatic activity $(3,6)$, a finding that has prompted investigators to test whether a loss of SOD1 activity can kill neurons. It was unequivocally shown that reducing SOD1 activity to about $50 \%$ using antisense 
oligonucleotides kills pheochromocytoma-12 (PC-12) cells and motor neurons in spinal cord organotypic cultures $(7,8)$. However, mutant mice deficient in SOD1 do not develop any motor neuron disease (9), and the transgenic expression of different SOD1 mutants in both mice (10-12) and rats (13) causes an ALS-like syndrome in these animals, whether SOD1 free radical-scavenging catalytic activity is increased, normal, or almost absent $(10-14)$. These observations provide compelling evidence that the cytotoxicity of mutant SOD1 is mediated not by a loss-of-function but rather by a gain-of-function effect (15).

\section{Transgenic mutant SOD1 mouse model of ALS}

As indicated above, the transgenic expression of different SOD1 mutants in both mice (10-12) and rats (13) produces a paralytic syndrome in these animals that replicates the clinical and pathological hallmarks of ALS. The age at onset of symptoms and the lifespan of these transgenic rodents vary among the different lines, depending on the mutation expressed and its level of expression, but when they become symptomatic they invariably show motor abnormalities that progress with the same pattern $(11,16)$.

The first motor abnormality, at least in mice, is the development of a fine tremor in at least one limb when the animal is held in the air by the tail (16). Thereafter, weakness and atrophy of proximal muscles, predominantly in the hind limbs, develop progressively. At the end stage, transgenic mutant SOD1 mice are severely paralyzed and can no longer feed or drink on their own (16). Neither their nontransgenic littermates nor agematched transgenic mice expressing wild-type SOD1 enzyme develop any of these motor abnormalities.

The first neuropathological changes seen in transgenic mutant SOD1 mice are perikarya, axonal and dendritic vacuoles in motor neurons with little involvement in the surrounding neuropil, and undetectable neuronal loss or gliosis $(11,17)$. In the transgenic mutant SOD1 mice that express a glycine-to-alanine substitution at position 93 (G93A) (10), these changes are observed in 4- to 6-week-old asymptomatic animals. By the time the first symptom, fine limb tremor, arises (about 90 days), vacuolization is prominent, and some neuronal loss, especially of large motor neurons $(>25$ $\mu \mathrm{m})$, is observed in the spinal cord. At the end stage, dramatic paralysis (about 140 days), there is still some degree of vacuolization, but the prominent features are the dramatic loss of motor neurons $(-50 \%)$, an abundance of dystrophic neurites, a marked gliosis (18), some globular Lewy body-like intracellular inclusions, and a dearth of motor neurons filled with phosphorylated neurofilaments $(10,16,17,19)$. Despite the close similarities between the phenotype of transgenic mutant SOD1 rodents and ALS, this experimental model departs from the human disease in a few important ways. First, vacuolar degeneration has not been a well-recognized component of motor neuron pathology in ALS. Second, neurofilamentous accumulation in cell bodies and proximal axons is infrequent in the lines of transgenic animals that express mutant SOD1, while it is conspicuous in ALS. Third, none of the transgenic lines show degeneration in the rodent equivalent of the human corticospinal tract. Notably, these transgenic animals replicate in rodents the effect of mutant SOD1, but how relevant this is to the sporadic form of ALS - which is not linked to SOD1 mutations - is unknown. Despite these imperfections and limitations, transgenic mutant SOD1 rodents unquestionably represent an excellent experimental model of ALS, one which has already generated valuable insights into the pathogenesis of ALS and opened new therapeutic avenues for this dreadful disease.

\section{Hypothesis for mutant SOD1 cytotoxicity}

Despite the explosion of ALS research engendered by the discovery of the SOD1 mutations, the actual nature of the gained function by which mutant SOD1 kills motor neurons in ALS remains elusive. Multiple mechanisms have been implicated in the demise of motor neurons in ALS (20), but only a few may be directly relevant to the form linked to mutant SOD1. For instance, the known free radical-scavenging function of SOD1 led researchers to believe that mutant SOD1-induced neurodegeneration was due to an oxidative stress. This idea was, at least initially, received with enthusiasm due to the fact that a variety of markers of oxidative damage are indeed increased in ALS spinal cords (20). Currently, it is thought that, if SOD1 mutants were to generate oxidative stress, they could do so by two distinct and not mutually exclusive mechanisms. In the first mechanism, the point mutations would relax SOD1 conformation, hence allowing abnormal kinds or amounts of substrates to reach and react with the transitional metal - copper - contained in the catalytic site of the enzyme. Among the aberrant substrates to be proposed are peroxynitrite (21) and hydrogen peroxide (22), both of which can directly or indirectly mediate serious tissue damage. In the second mechanism, it is speculated that SOD1 mutations are associated with a labile binding of zinc to the protein (23), and that, by having lost zinc, mutant SOD1, in the presence of nitric oxide, will catalyze the production of peroxynitrite (24), which can inflict serious oxidative damage to virtually all cellular elements.

Alternatively, mutant SOD1 cytotoxicity may result from the propensity of this mutant protein to form intracellular proteinaceous aggregates (25), which are a prominent pathological feature of several of the transgenic lines $(12,13,19)$, and of various cultured cell types expressing mutant SOD1, including motor neurons (26). As in other neurodegenerative disorders with intracellular inclusions, whether or not these proteinaceous aggregates are actually noxious remains uncertain. Nevertheless, it may be speculated that their presence in the cytosol of motor neurons may be deleterious, by, for example, impairing the microtubule-dependent axonal transport of vital nutriments, or by perturbing the normal turnover of intracellular proteins (27).

Early on in the effort to determine the nature of mutant SOD1's gained function, it was discovered that transfected neuronal cells expressing mutant 
SOD1 cDNA were dying by apoptosis (28), a form of programmed cell death (PCD). Similar observations were subsequently made in transfected PC-12 cells (29) and in primary neurons grown from transgenic mice expressing mutant SOD1 (30). Collectively, these in vitro data have led many investigators to consider that mutant SOD1 may kill motor neurons by activating PCD, a term that we here use in the sense of cell death mediated by specific signaling pathways. The possible implication of PCD in ALS has been rather appealing to the field of motor neuron diseases ever since the neuronal apoptosis inhibitory protein (NAIP) was identified as a candidate gene for an inherited ALSrelated disorder, spinal muscular atrophy (31). The remainder of this review will focus on PCD in ALS. Because most of the published mechanistic investigations of that topic have been performed in transgenic mutant SOD1 mice, this appraisal will emphasize this mouse model of ALS, but human data will be cited whenever possible to support the relevance of the animal findings to the human condition.

\section{Morphology of dying motor neurons}

In light of the presumed proapoptotic properties of mutant SOD1 observed in vitro, it may be wondered whether, in transgenic mutant SOD1 mice, dying spinal cord motor neurons would also exhibit features of apoptosis, whose morphological hallmarks include cytoplasmic and nuclear condensation, compaction of nuclear chromatin into sharply circumscribed masses along the inside of the nuclear membrane, and structural preservation of organelles (at least until the cell is broken into membrane-bound fragments called apoptotic bodies that are phagocytized). This question has been examined in several careful morphological studies performed in transgenic mutant SOD1 mice $(17,32-34)$. In these animals, most of the sick neurons are atrophic, and their cytoplasm is occupied with vacuoles corresponding to dilated rough ER, Golgi apparatus, and mitochondria (17). From our own ultrastructural studies in these mice (S. Przedborski, unpublished observations), we can add that many sick neurons have diffusely condensed cytoplasm and nuclei and irregular shapes. Although the actual type of this cell death remains to be determined, these dying neurons exhibit a rather nonapoptotic morphology with some features reminiscent of autophagic or cytoplasmic neuronal death (35). Yet, in our experience, definitely apoptotic cells are seen but are rare in the spinal cord of affected transgenic mutant SOD1 mice (Figure 1a). For instance, in end-stage transgenic SOD1G93A mice, which have lost about $50 \%$ of their anterior-horn motor neurons, it can be estimated that about two apoptotic cells will be seen per $40-\mu \mathrm{m}$-thick section of the lumbar spinal cord. We have also observed that the vast majority of these apoptotic cells no longer exhibit definite morphological characteristics or express phenotypic markers that allow their identification as neurons or glia. However, some (less than $15 \%$ ) of the spinal cord apoptotic cells are still immunoreactive for specific proteins such as neurofilament or glial fibrillary acid protein (33), suggesting that both neuronal and glial cells are dying by apoptosis in the mutant SOD1 model (34). In our opinion, the paucity of apoptotic dying motor neurons in this mouse model of ALS reflects the difficulty in detecting these cells by morphological means due to the presumed low daily rate of motor neuron loss (16) and the notoriously rapid disappearance of apoptotic cells. Forms of PCD with morphological features distinct from apoptosis also exist (35-37), making it difficult to exclude the possibility that a nonapoptotic form of PCD underlies mutant SOD1-related cellular degeneration. a

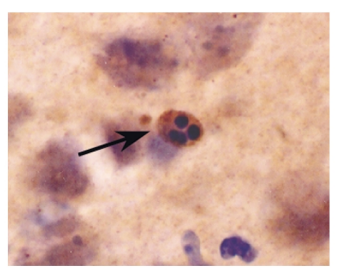

C

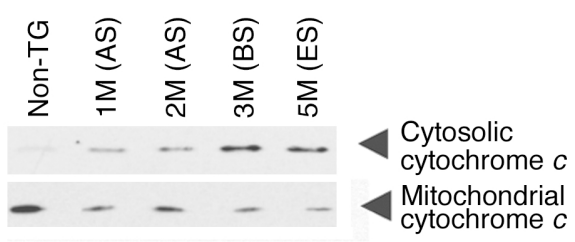

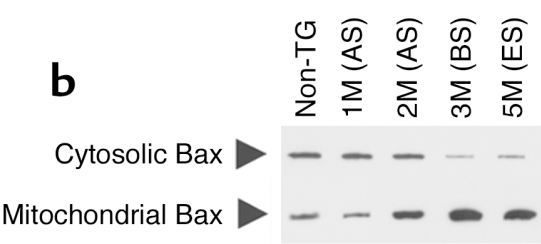
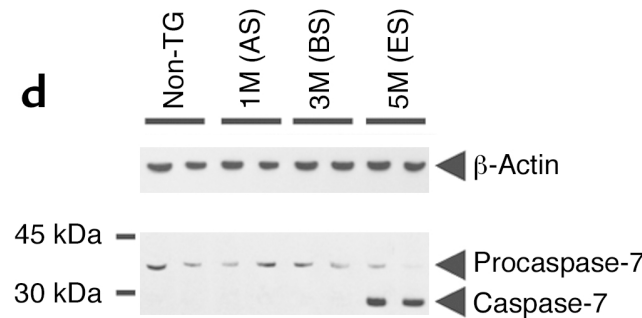

\section{Figure 1}

Illustrations of PCD alterations in spinal cord of transgenic mutant SOD1 mice. (a) Photomicrograph of definitely apoptotic cells found in the anterior horn of an end-stage transgenic SOD1 ${ }^{\mathrm{G} 93 \mathrm{~A}}$ mouse. The arrow shows several typical round chromatin clumps. (b) Western blot analysis of spinal cord extracts, demonstrating the relocation of Bax from the cytosol (top) to the mitochondria (bottom) over the course of the disease. (c) Coincidental changes of cytochrome $c$ in the opposite direction. (d) Later in the disease, effector caspases such as caspase-7 are activated. $1 \mathrm{M}(\mathrm{AS})$ : 1 month, asymptomatic stage; $2 \mathrm{M}(\mathrm{AS}): 2$ months, asymptomatic stage; $3 \mathrm{M}$ (BS): 3 months, beginning of symptoms; $5 \mathrm{M}$ (ES): 5 months, end stage; Non-TG: nontransgenic littermates. Modified from ref. 63. 


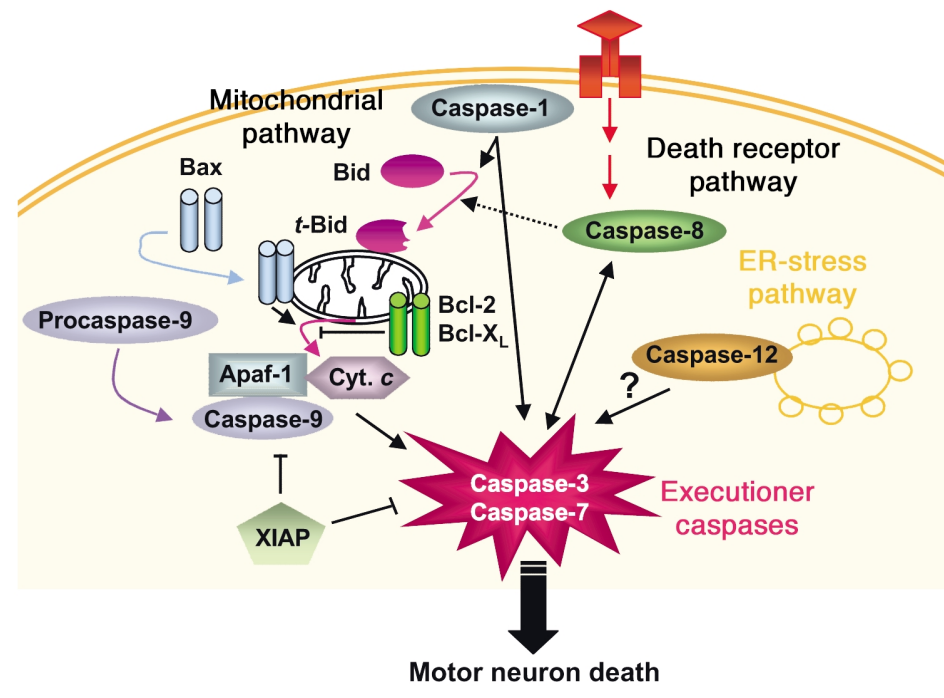

Figure 2

Molecular pathways of PCD. To date, at least three different PCD molecular pathways have been recognized: the mitochondrial pathway (also called intrinsic), the death receptor pathway (also called extrinsic), and the ER pathway. In the mitochondrial $\mathrm{PCD}$ pathway, translocation of the proapoptotic protein Bax and the $\mathrm{BH} 3$-domainonly protein Bid from the cytosol to the mitochondria promotes cell death by inducing the release of cytochrome $c$ (Cyt. $c$ ) from the mitochondria to the cytosol; although both full-length and truncated Bid ( $t$-Bid) translocate to the mitochondria, $t$-Bid is the most biologically active form. Once in the cytosol, cytochrome $c$ activates caspase9 in the presence of Apaf- 1 , which, in turn, activates downstream executioner caspases. This pathway can be inhibited by the antiapoptotic protein $\mathrm{Bcl}-2$ and by the protein caspase inhibitor X chromosome-linked inhibitor of apoptosis (XIAP). In the death receptor pathway, caspase- 8 is activated by death receptors (members of the TNFR family) in the plasma membrane via the intermediary of adapter proteins. Death receptors include Fas (CD95) and the low-affinity neurotrophin receptor (p75 NTR). Activated caspase- 8 then activates executioner caspases, directly or indirectly, through the activation of Bid. Stress in the ER, including disruption of ER-calcium homeostasis and accumulation of excess proteins in the ER, can also result in apoptosis through activation of caspase-12. This caspase is not activated by membrane- or mitochondria-targeted apoptotic signals. Activation of the upstream caspase-1, the key enzyme responsible for the activation of IL-1, also results in activation of executioner caspases and enhances, at least in part through the cleavage of Bid, the activation of the mitochondria-dependent apoptotic pathway.

In human ALS cases, using morphological criteria including size, shape, and aggregates of Nissl substance, Martin (38) has arranged residual spinal cord motor neurons in ALS postmortem samples in three categories that he believes reflect different stages of degeneration. In the chromatolysis stage, motor neurons still resemble their normal counterparts except that the cell body appears swollen and round, the Nissl substance dispersed, and the nucleus eccentrically placed. Some chromatolytic neurons have prominent cytoplasmic hyaline body inclusions. In the attritional stage, the cytoplasm and the nucleus appear homogenous and condensed, and the cell body appears shrunken and with hazy multipolar shape. In the so-called apoptotic stage, the affected motor neuron is approximately one-fifth of its normal diameter, the cytoplasm and nucleus are extremely condensed, and the cell body adopts a fusiform or round shape devoid of any process. Notably, in none of the three stages do residual motor neurons show appreciable cytoplasmic vacuoles or nuclear condensation accompanied by round chromatin clumps. Taken together, these findings suggest that, while degenerating neurons in both human ALS and its experimental models do exhibit some features reminiscent of apoptosis, the vast majority of dying cells cannot confidently be labeled as typical apoptotic.

\section{Expression of apoptotic markers}

Besides exhibiting singular morphological features, apoptotic cells may also show a variety of cellular alterations. The detection of internucleosomal DNA cleavage by either gel electrophoresis or in situ methods has emerged as a popular means of supporting the occurrence of apoptosis in all sorts of pathological situations, including ALS. However, like many of these apoptotic markers, DNA fragmentation detected by in situ methods (e.g., terminal deoxynucleotidyl transferasemediated nick end labeling) is now well recognized as also occurring in nonapoptotic cell death, including necrosis (35). So the value of DNA cleavage evidenced by in situ techniques as a specific marker of apoptosis may be limited. In addition to this caveat, the search for DNA fragmentation in ALS postmortem samples has generated conflicting results. In one autopsy study, DNA fragmentation was detected by an in situ method in spinal cord motor neurons in ALS but not in control specimens (39). In two other similar studies, DNA fragmentation was detected not only in the motor cortex and spinal cord of ALS specimens, but also, though to a lesser degree, in control specimens $(40,41)$. In a subsequent study, internucleosomal DNA fragmentation was detected in affected (e.g., motor cortex and spinal cord) but not in spared brain regions (e.g., somatosensory cortex) of ALS cases (38), and, in diseased motor neurons, only at the somatodendritic attrition and apoptotic stages and not at the chromatolytic stage (38). The author of that study has also documented DNA fragmentation in anteriorhorn gray matter of the spinal cord and motor cortex of ALS cases by gel electrophoresis (38), a technique not frequently used in the nervous system to identify apoptosis since, in many neurological situations, it is difficult to obtain samples with a sufficiently high proportion of dying cells. In contrast to all these positive findings, other groups, using similar techniques and tissue samples, have failed to provide any evidence of internucleosomal cleavage of DNA in postmortem tissue from human ALS cases or from animal models of the disease $(32,41,42)$. Although the actual reason for these divergent results is unclear, they cast doubt on the reliability and even the specificity of such findings. 
Two other apoptotic markers, the $\mathrm{Le}^{\mathrm{Y}}$ antigen (43) and fractin (44), were also studied in ALS, and here the picture seems less ambiguous. Neither marker was detected in spinal cords of controls, but were highly expressed in spinal cords of, respectively, ALS cases (39) and transgenic SOD1 ${ }^{\mathrm{G} 93 \mathrm{~A}}$ mice (34). Likewise, the levels of the apoptosis-related protein prostate apoptosis response-4 (45) were increased in spinal cord samples from both ALS patients and transgenic mutant SOD1 mice compared with their respective controls (46). Together with the morphological data summarized above, these findings support the view that apoptosis occurs in ALS. What all of these studies fail to do, however, is to provide definite mechanistic insights into the significance of these alterations in the pathogenesis of ALS.

\section{Activation of apoptotic molecular pathways}

Given the ambiguous results of the morphological studies, it appears that a more convincing approach to evaluating the role of apoptosis in ALS may be to determine whether the neurodegenerative process in transgenic mutant SOD1 mice, irrespective of the morphology of the dying cells, involves known molecular mediators of PCD, and whether targeting such key factors can affect the course of the disease.

PCD is a multistep machinery (Figure 2) that involves a complex interaction between survival pathways, activated by trophic factors, and death pathways, activated by various stresses. So far, the two pathways that have been most implicated in neuronal survival are the PI3K pathway, which activates Akt (also known as protein kinase $\mathrm{B}$ ) to suppress the activation of proapoptotic proteins, and the extracellular signal-regulated kinase/MAPK (ERK/MAPK) pathway, which activates antiapoptotic proteins (47).

The best-known PCD-mediating pathways are those involved in the activation of caspase-3. The caspases are a family of cysteine-aspartate proteases (Figure 2; see below for details), many of which are involved in PCD either at the level of upstream signaling (notably caspase-8 and caspase-9) or more downstream at the effector level (notably caspase-3). Caspase-8 and caspase- 9 both cleave procaspase- 3 to activate it. Caspase- 9 is activated by a signal derived from mitochondria under the control of the Bcl-2 family of proteins (Figure 2; see below for details). Caspase- 8 is activated by death receptors (members of the TNF receptor family) in the plasma membrane via the intermediary of adapter proteins (48). Death receptors include the low-affinity neurotrophin receptor (p75 ${ }^{\mathrm{NTR}}$ ) and Fas (CD95); the latter seems to participate in the death of embryonic motor neurons in primary cultures (49), but whether Fas contributes to the death of mature motor neurons and to the neurodegenerative process in transgenic mice expressing mutant SOD1 remains to be demonstrated. Other key molecules in PCD signaling include ceramide, MAPKs (JNK and p38), and the transcription factors activator protein- 1 and NF- $\mathrm{KB}(47,48)$.

In light of the presumed proapoptotic properties of mutant SOD1 (28), it is tempting to suggest that the mutant protein may be a death-signaling molecule in itself, either directly, by setting in motion the PCD cascade, or indirectly, by interacting with a variety of intracellular targets such as trophic factors, Bcl-2 family members, or even mitochondria. Mitochondria are a particularly appealing target, because they not only contain mutant SOD1 (50) but are structurally and functionally altered in transgenic mutant SOD1 mice $(51,52)$, and because they play a pivotal role in PCD (53). Also relevant to the issue of death and survival signals in the mutant SOD1 model are the Western blot and immunohistochemical demonstrations of the weakening surviving signal mediated by PI3K/Akt in spinal cords of transgenic mutant SOD1 mice even before overt neuropathological features arise (54). Once the mutant SOD1-mediated neurodegenerative process has been initiated, several secondary alterations develop in spinal cords of transgenic mutant SOD1 mice, including microglial cell activation (18) and T cell infiltration (55), both of which may release a plethora of cytokines and other pro-PCD mediators. Accordingly, while the nature of the initial death signal in transgenic mutant SOD1 mice remains elusive, in a more advanced stage of the disease the increased expression of several extracellular inflammation-related factors such as IL-1 $\beta$, IL-6, and TNF- $\alpha$ (56) may amplify the death signals that are already reaching motor neurons in this mouse model of ALS, by activating death receptors such as Fas (49). IL- $1 \beta$ content is also elevated in human ALS spinal cords (57).

\section{The role of the Bcl-2 family in motor-neuronal cell death in ALS}

The Bcl-2 family, implicated in the regulation of PCD (Figure 2), is composed of both cell-death suppressors such as Bcl-2 and Bcl- $\mathrm{X}_{\mathrm{L}}$ and promoters such as Bax, $\mathrm{Bad}, \mathrm{Bak}$, and $\mathrm{Bcl}-\mathrm{xS}$ (58). Many of these molecules are present and active within the nervous system and appear to be potent modulators of neuronal death. In human ALS cases and affected transgenic SOD1G93A mice, Bcl-2 mRNA content appears significantly decreased and Bax mRNA content significantly increased in the lumbar cord compared with those of controls $(59,60)$. This is consistent with the finding in both human ALS cases and symptomatic transgenic SOD $1^{\mathrm{G} 93 \mathrm{~A}}$ mice that the spinal cord expressions of the antiapoptotic proteins $\mathrm{Bcl}-2$ and $\mathrm{Bcl}-\mathrm{X}_{\mathrm{L}}$ are either unchanged $(40,61)$ or decreased $(38,60)$, whereas that of the proapoptotic Bax and Bad proteins is increased $(38,40,60)$. Different SOD1 mutations do not cause exactly the same neuropathology. It is important to note that a very similar pattern of changes of selected pro- and anti-cell death Bcl-2 family members was found in spinal cords of affected transgenic SOD 1 G86R mice compared with their wild-type counterparts (62). None of these alterations, however, is seen in young asymptomatic transgenic SOD1 ${ }^{\mathrm{G} 93 \mathrm{~A}}$ mice; but they clearly become progressively more conspicuous as the neurodegenerative process progresses (60). With regard to function, in the spinal cords of both ALS patients and affected transgenic mice expressing mutant SOD1, Bax is not only upregulated but is also 
expressed mainly in its active homodimeric conformation $(38,60)$. As illustrated in Figure $1 \mathrm{~b}$, it is markedly relocated from the cytosol to the mitochondria $(38,63)$; this relocation is, in many cellular settings, a prerequisite to the recruitment of the mitochondria-dependent apoptosis pathway. So it seems that, in ALS, during the neurodegenerative process, the fine-tuned balance between cell-death antagonist and agonist of the Bcl-2 family is upset in favor of pro-cell death forces. In support of this view is the finding that overexpression of Bcl-2, presumably by buffering some of the pro-cell death drive (60), mitigates neurodegeneration and prolongs survival in transgenic SOD $1^{\mathrm{G} 93 \mathrm{~A}}$ mice (64); a similar beneficial effect of Bcl-2 was reported in mutant SOD1-transfected PC-12 cells (29).

Other meaningful Bcl-2 family members that appear to be in play in ALS include Bid and Harakiri, two potent pro-PCD peptides, which can participate in the cell death process, either directly or indirectly, by potentiating the effect of Bax. Bid appears to be highly expressed in the spinal cord of transgenic SOD $1^{\mathrm{G} 93 \mathrm{~A}}$ mice and is cleaved into its most active form during the progression of the disease (65). Harakiri's expression has been detected in motor neurons of ALS, but not of control, spinal cord specimens, specifically in spared neurons, of which some exhibited an abnormal morphology reminiscent of that labeled by Troost et al. (61) as apoptotic (66).

The quest to elucidate how Bcl-2 family members are deregulated in ALS is fascinating. As in other pathological situations, it is unlikely that mutant SOD1 directly produces the observed changes in Bax. It is more likely that mutant SOD1 ignites intracellular signaling pathways, which, in turn, cause Bax upregulation and translocation. This scenario would be consistent with what we currently know about the regulation of Bax and how Bax is usually brought into action in PCD. The tumor suppressor protein p53 counts among the rare molecules known to regulate Bax expression (67). In normal situations, p53 basal levels in the cell are very low, but upon activation, as seen in pathological situations, there is a rapid rise in p53 mRNA and protein levels, as well as posttranslational modifications that stabilize the protein (68). Activation of the $\mathrm{p} 53$ pathway in ALS is evidenced by the demonstration that $\mathrm{p} 53$ is increased in the nuclear fraction of affected brain regions in ALS patients (69), as is p53 immunostaining in neuron nuclei of transgenic SOD1 ${ }^{\mathrm{G} 86 \mathrm{R}}$ mice (62). Despite the compelling evidence that p53 is activated in ALS, two independent studies have failed to provide any supportive data for an actual role for this transcriptional factor in mutant SOD1-mediated neurodegeneration $(70,71)$.

\section{Caspases in the ALS neurodegenerative process}

Caspases are members of a distinct family of cysteine proteases that share the ability to cleave their substrates after specific aspartic acid residues and that are present in cells as inactive zymogens, called procaspases. So far, 14 different mammalian caspases have been identified that differ in primary sequence and substrate specificity.
An instrumental role for caspases in ALS neurodegeneration is supported by the demonstration that the irreversible broad-caspase inhibitor benzyloxycarbonylVal-Ala-Asp (O-methyl)-fluoromethylketone attenuates mutant SOD1-mediated cell death in transfected PC-12 cells (29) and in transgenic SOD $1^{\mathrm{G} 93 \mathrm{~A}}$ mice (57).

All of the identified caspases are grouped based on their function. One group includes caspases $-1,-4,-5$, $-11,-12$, and -14 , which are now believed to play a primary role in cytokine maturation. Among these, in ALS, the lion's share of attention until now has been given to caspase-1, the key enzyme responsible for the activation of IL-1. Procaspase-1 is highly expressed in spinal cord motor neurons, and its activation in the spinal cord of transgenic mutant SOD1 mice coincides with the development of the glial response and with the very beginning of the loss of motor neurons $(33,34,57$, 63,72 ). Despite caspase-1's likely indirect role in PCD, chronic inhibition of caspase- 1 by a dominant negative mutant of the enzyme has been proven effective in prolonging the life of transgenic SOD1 $1^{\mathrm{G} 93 \mathrm{~A}}$ mice (73). So far, the status of the other members of the caspase- 1 subfamily in ALS is unknown. Some preliminary investigations show that caspase-12, which is known to be activated following ER stress (74), is expressed in motor neurons of nontransgenic mice, and even more so in those of symptomatic transgenic SOD $1^{\mathrm{G} 93 \mathrm{~A}}$ mice (C. Guégan et al., unpublished observations). In symptomatic transgenic SOD $1^{\mathrm{G} 93 \mathrm{~A}}$ mice, most of the motor neurons immunopositive for caspase-12 appear condensed, shrunken, and vacuolized. Although more work on caspase-12 remains to be done in this model of ALS, our preliminary data argue that sick cells are the site of an ER stress whose occurrence could well contribute to the overall cascade of deleterious events that ultimately underlies the demise of spinal cord motor neurons in the mutant SOD1 model.

By contrast, caspases $-2,-3,-6,-7,-8,-9$, and -10 have been implicated in apoptosis per se, although their roles can be further divided into "initiator" and "effector."

Initiator caspases include procaspases $-2,-8,-9$, and -10 , all of which have long prodomains and proteinprotein interaction motifs, such as the death-effector domain and the caspase-activation and -recruitment domain, that contribute to the transduction of various signals into proteolytic activity. Procaspase-8 is activated after ligation of certain cell surface receptors, such as the TNF receptors. Interestingly, while significant glial response and production of IL- $1 \beta$ occur early in transgenic mutant SOD1 mice (see above), activation of procaspase- 8 , like induction of TNF- $\alpha$ (56), is only detected in spinal cords near the end stage (65). This suggests that, in this ALS model, the TNF/caspase- 8 machinery may be a late contributor to the degenerative process. Caspase- 2 is another initiator of PCD whose activation occurs in the spinal cord of affected transgenic mutant SOD1 mice (S. Vukosavic et al., unpublished observations). Yet ablation of caspase-2 in transgenic SOD $1^{\mathrm{G} 93 \mathrm{~A}}$ mice has been reported to be of no consequence to the expression of the disease (75), indicating that whatever the role of caspase- 2 is in 
ALS, it is dispensable. A third caspase initiator is caspase-9, whose role is pivotal in the so-called mitochondria-dependent PCD pathway (53). Here, after a death stimulus, released mitochondrial cytochrome $c$ interacts in the cytosol with apoptotic protease-activating factor-1 (Apaf-1) in the presence of dATP, which stimulates the processing of procaspase- 9 into its active form, which in turn can activate the downstream executioner caspases (see below). Evidence of prominent recruitment of this mitochondrial pathway has been documented in spinal cord specimens of both ALS patients and transgenic SOD $1^{\mathrm{G} 93 \mathrm{~A}}$ mice (63). In that study, it is shown that, while cytochrome $c$ is confined to the mitochondria in cells in the control samples, it is diffusely distributed in the cytosol in several of the spared cells, especially neurons, in the pathological samples. It is also demonstrated, at least in transgenic mutant SOD1 ${ }^{\mathrm{G} 93 \mathrm{~A}}$ mice, that the mitochondrial cytochrome $c$ translocation to the cytosol occurs at the same time as the cytosolic Bax translocation to the mitochondria and activation of procaspase-9, and before activation of downstream caspase executioners such as procaspase- 3 and procaspase-7 (Figure 1, b-d). Because caspase- 9 is thought to be so critical in many cell-death settings, it is very likely that the observed translocation of cytochrome $c$ and activation of procaspase-9 in ALS represent significant pathological events. Consistent with this view is the finding that prevention of mitochondrial cytochrome $c$ release lengthens the lifespan of transgenic SOD1G93A mice (76).

Effector caspases include procaspases $-3,-6$, and -7 , all of which have short prodomains and lack intrinsic enzymatic activity. However, upon their cleavage, which is triggered by, for example, initiator caspases, effector caspases acquire the capacity to cleave a large number of intracellular substrates, which probably results in the eventual death of the cell. Consistent with this scenario, it has been reported that key effector caspases such as caspase- 3 and caspase-7 (see Figure $1 \mathrm{~d}$ ) are indeed activated in spinal cords of transgenic mutant SOD1 mice in a time-dependent manner that parallels the time course of the neurodegenerative process $(33,34)$; activation of procaspase- 3 has also been observed in spinal cord samples from ALS patients (38). Yet current data on the sequence of events in the PCD cascade indicate that, once effector caspases have been activated, the cell death process, at least in certain pathological settings, has reached a point of no return. This would suggest that, in these specific conditions, the death commitment point is situated upstream of these caspases, and, consequently, interventions aimed at inhibiting these downstream caspases may fail to provide any real neuroprotective benefit (77). Whether this applies to the demise of motor neurons in ALS remains to be determined.

\section{Conclusion}

In this review we have described evidence that numerous key molecular components of PCD are recruited in ALS. We have also shown that, while precious data on PCD in ALS have been obtained thanks to the study of postmortem human samples, information regarding the temporal relationships of these changes and their significance in the pathological cascade emanates essentially from the use of transgenic mutant SOD1 mouse models. In light of the abovedescribed PCD-related changes, it would appear that this active form of cell death is not the sole pathological mediator of cell demise in ALS but rather one key component within a coalition of deleterious factors ultimately responsible for the degenerative process. As discussed above, however, the actual relationships between mutant SOD1 and the various other presumed culprits represented by protein aggregates, oxidant production, and PCD activation are still unknown, and a better understanding of the pathogenic cascade in ALS will require their elucidation.

In our opinion, one of the most important takehome messages from the body of work summarized above is that an apoptotic morphology should not be used as the sole criterion of whether molecular pathways of PCD have been recruited. Indeed, we can not stress enough that the PCD molecular pathways may be activated in a neurodegenerative process such as that seen in ALS, even when the prevalent morphology of the dying cells is nonapoptotic. Relatedly, caspase-9 is instrumental in paraptosis (37), a specific morphological form of nonapoptotic cell death.

Apart from the question of whether the morphology of dying neurons in ALS is apoptotic, but still relevant to our discussion, is the contrast between the paucity of morphologically identified dying cells and the rather robust spinal cord molecular PCD alterations. How can this striking discrepancy be reconciled? First, it is possible that the morphological expression of PCD is much more ephemeral than its molecular translation. Therefore, since in ALS the degenerative process is asynchronous, small lasting differences in the expression of these markers may have significant impact on the total number of cells that exhibit a given marker at a given time point. Second, it is also possible that, since apoptotic morphological features are confined to the cell body while PCD molecular alterations may be found not only in cell but also in cell processes, axons, and nerve terminals. Thus, the detection of PCD morphology may be much more challenging than the detection of PCD molecular events. Third, the molecular tools used in all of the cited studies see not only the rare cells that are truly dying but also the numerous sick cells that may or may not ultimately die and that thus may or may not show the typical apoptotic morphology.

Another important point that derives from the work in transgenic mutant SOD1 mice is that not only neurons but also glial cells appear to be the site of PCD-cascade activation. This observation does not undermine the potential pathogenic role of PCD in the ALS death process, but it raises the possibility that PCD may not only kill neurons in this disease. However, since SOD1 is expressed in all cells, not only in motor neurons, it is possible that the activation of PCD in both neurons and glia reflects the ubiquitous nature of the mutant protein expression. 
Whether PCD is also activated in neuron and glial cells in the forms of ALS that are not linked to mutant SOD1 is unknown at this point.

Clearly, the overall mechanism of neurodegeneration in ALS is still incompletely known. Nevertheless, the available evidence indicates that PCD is in play in ALS and thus warrants further investigation of the role of the PCD cascade in ALS pathogenesis and treatment. The most effective therapeutic strategies tested so far in transgenic mutant SOD1 mice target very distinct molecular pathways. We can therefore imagine that, ultimately, the best therapy for ALS will come from a combination of several interventions and not from a single treatment. In keeping with this view, unraveling the sequence of key PCD factors recruited during ALS neurodegeneration should enable us to identify the most significant molecules to be targeted by this therapeutic cocktail to produce optimal neuroprotection.

\section{Acknowledgments}

The authors wish to thank Robert E. Burke and Miquel Vila for their insightful comments on the manuscript, and Pat White and Brian Jones for their help in its preparation. The authors also acknowledge the support of National Institute of Neurological Disorders and Stroke grants R29 NS37345, RO1 NS38586, NS42269, and P50 NS38370, US Department of Defense grant DAMD 17-99-1-9471, the Lowenstein Foundation, the Lillian Goldman Charitable Trust, the Parkinson's Disease Foundation, the Muscular Dystrophy Association, the ALS Association, and Project ALS.

1. Rowland, L.P. 1995. Hereditary and acquired motor neuron diseases. In Merritt's textbook of neurology. L.P. Rowland, editor. Williams \& Wilkins. Philadelphia, Pennsylvania, USA. 742-749.

2. Brown, R.H., Jr. 1995. Amyotrophic lateral sclerosis: recent insights from genetics and transgenic mice. Cell. 80:687-692.

3. Deng, H.-X., et al. 1993. Amyotrophic lateral sclerosis and structural defects in $\mathrm{Cu}, \mathrm{Zn}$ superoxide dismutase. Science. 261:1047-1051.

4. Rosen, D.R., et al. 1993. Mutations in $\mathrm{Cu} / \mathrm{Zn}$ superoxide dismutase gene are associated with familial amyotrophic lateral sclerosis. Nature. 362:59-62.

5. Cudkowicz, M.E., et al. 1997. Epidemiology of mutations in superoxide dismutase in amyotrophic lateral sclerosis. Ann. Neurol. 41:210-221.

6. Przedborski, S., et al. 1996. Blood superoxide dismutase, catalase and glutathione peroxidase activities in familial and sporadic amyotrophic lateral sclerosis. Neurodegeneration. 5:57-64.

7. Troy, C.M., and Shelanski, M.L. 1994. Down-regulation of copper/zinc superoxide dismutase causes apoptotic death in PC12 neuronal cells. Proc. Natl. Acad. Sci. USA. 91:6384-6387.

8. Rothstein, J.D., Bristol, L.A., Hosler, B., Brown, R.H., Jr., and Kuncl, R.W. 1994. Chronic inhibition of superoxide dismutase produces apoptotic death of spinal neurons. Proc. Natl. Acad. Sci. USA. 91:4155-4159.

9. Reaume, A.G., et al. 1996. Motor neurons in $\mathrm{Cu} / \mathrm{Zn}$ superoxide dismutase-deficient mice develop normally but exhibit enhanced cell death after axonal injury. Nat. Genet. 13:43-47.

10. Gurney, M.E., et al. 1994. Motor neuron degeneration in mice that express a human $\mathrm{Cu}, \mathrm{Zn}$ superoxide dismutase mutation. Science. 264:1772-1775.

11. Wong, P.C., et al. 1995. An adverse property of a familial ALS-linked SOD1 mutation causes motor neuron disease characterized by vacuolar degeneration of mitochondria. Neuron. 14:1105-1116.

12. Bruijn, L.I., et al. 1997. ALS-linked SOD1 mutant G85R mediated damage to astrocytes and promotes rapidly progressive disease with SOD1containing inclusions. Neuron. 18:327-338.

13. Nagai, M., et al. 2001. Rats expressing human cytosolic copper-zinc superoxide dismutase transgenes with amyotrophic lateral sclerosis: associated mutations develop motor neuron disease. J. Neurosci. 21:9246-9254.

14. Subramaniam, J.R., et al. 2002. Mutant SOD1 causes motor neuron disease independent of copper chaperone-mediated copper loading. Nat. Neurosci. 5:301-307.
15. Brown, R.H., Jr. 1995. Superoxide dismutase in familial amyotrophic lateral sclerosis: models for gain of function. Curr. Opin. Neurobiol. 5:841-846.

16. Chiu, A.Y., et al. 1995. Age-dependent penetrance of disease in a transgenic mouse model of familial amyotrophic lateral sclerosis. Mol. Cell. Neurosci. 6:349-362.

17. Dal Canto, M.C., and Gurney, M.E. 1995. Neuropathological changes in two lines of mice carrying a transgene for mutant human $\mathrm{Cu}, \mathrm{Zn} \mathrm{SOD}$, and in mice overexpressing wild type human SOD: a model of familial amyotrophic lateral sclerosis (FALS). Brain Res. 676:25-40.

18. Almer, G., Vukosavic, S., Romero, N., and Przedborski, S. 1999. Inducible nitric oxide synthase upregulation in a transgenic mouse model of familial amyotrophic lateral sclerosis. J. Neurochem. 72:2415-2425.

19. Tu, P.H., et al. 1996. Transgenic mice carrying a human mutant superoxide dismutase transgene develop neuronal cytoskeletal pathology resembling human amyotrophic lateral sclerosis lesions. Proc. Natl. Acad. Sci. USA. 93:3155-3160.

20. Cleveland, D.W., and Rothstein, J.D. 2001. From Charcot to Lou Gehrig: deciphering selective motor neuron death in ALS. Nat. Rev. Neurosci. 2:806-819.

21. Beckman, J.S., Carson, M., Smith, C.D., and Koppenol, W.H. 1993. ALS, SOD and peroxynitrite. Nature. 364:584.

22. Wiedau-Pazos, M., et al. 1996. Altered reactivity of superoxide dismutase in familial amyotrophic lateral sclerosis. Science. 271:515-518.

23. Crow, J.P., Sampson, J.B., Zhuang, Y.X., Thompson, J.A., and Beckman, J.S. 1997. Decreased zinc affinity of amyotrophic lateral sclerosis-associated superoxide dismutase mutants leads to enhanced catalysis of tyrosine nitration by peroxynitrite. J. Neurochem. 69:1936-1944.

24. Estevez, A.G., et al. 1999. Induction of nitric oxide-dependent apoptosis in motor neurons by zinc-deficient superoxide dismutase. Science. 286:2498-2500.

25. Bruijn, L.I., et al. 1998. Aggregation and motor neuron toxicity of an ALS-linked SOD1 mutant independent from wild-type SOD1. Science. 281:1851-1854.

26. Durham, H.D., Roy, J., Dong, L., and Figlewicz, D.A. 1997. Aggregation of mutant $\mathrm{Cu} / \mathrm{Zn}$ superoxide dismutase proteins in a culture model of ALS. J. Neuropathol. Exp. Neurol. 56:523-530.

27. Johnson, W.G. 2000. Late-onset neurodegenerative diseases: the role of protein insolubility. J. Anat. 196:609-616.

28. Rabizadeh, S., et al. 1995. Mutations associated with amyotrophic lateral sclerosis convert superoxide dismutase from an antiapoptotic gene to a proapoptotic gene: studies in yeast and neural cells. Proc. Natl. Acad. Sci. USA. 92:3024-3028.

29. Ghadge, G.D., et al. 1997. Mutant superoxide dismutase-1-linked familial amyotrophic lateral sclerosis: molecular mechanisms of neuronal death and protection. J. Neurosci. 17:8756-8766.

30. Mena, M.A., et al. 1997. Effects of wild-type and mutated copper/zinc superoxide dismutase on neuronal survival and L-DOPA-induced toxicity in postnatal midbrain culture. J. Neurochem. 69:21-33.

31. Roy, N., et al. 1995. The gene for neuronal apoptosis inhibitory protein is partially deleted in individuals with spinal muscular atrophy. Cell. 80:167-178.

32. Migheli, A., et al. 1999. Lack of apoptosis in mice with ALS. Nat. Med. 5:966-967.

33. Pasinelli, P., Houseweart, M.K., Brown, R.H., Jr., and Cleveland, D.W. 2000. Caspase- 1 and -3 are sequentially activated in motor neuron death in $\mathrm{Cu}, \mathrm{Zn}$ superoxide dismutase-mediated familial amyotrophic lateral sclerosis. Proc. Natl. Acad. Sci. USA. 97:13901-13906.

34. Vukosavic, S., et al. 2000. Delaying caspase activation by Bcl-2: a clue to disease retardation in a transgenic mouse model of amyotrophic lateral sclerosis. J. Neurosci. 20:9119-9125.

35. Clarke, P.G.H. 1999. Apoptosis versus necrosis. In Cell death and diseases of the nervous system. V.E. Koliatsos and R.R. Ratan, editors. Humana Press. Totowa, New Jersey, USA. 3-28.

36. Yaginuma, H., et al. 1996. A novel type of programmed neuronal death in the cervical spinal cord of the chick embryo. J. Neurosci. 16:3685-3703.

37. Sperandio, S., de Belle, I., and Bredesen, D.E. 2000. An alternative, nonapoptotic form of programmed cell death. Proc. Natl. Acad. Sci. USA. 97:14376-14381.

38. Martin, L.J. 1999. Neuronal death in amyotrophic lateral sclerosis is apoptosis: possible contribution of a programmed cell death mechanism. J. Neuropathol. Exp. Neurol. 58:459-471.

39. Yoshiyama, Y., Yamada, T., Asanuma, K., and Asahi, T. 1994. Apoptosis related antigen, $\mathrm{Le}(\mathrm{Y})$ and nick-end labeling are positive in spinal motor neurons in amyotrophic lateral sclerosis. Acta Neuropathol. (Berl.) 88:207-211.

40. Ekegren, T., Grundstrom, E., Lindholm, D., and Aquilonius, S.M. 1999. Upregulation of Bax protein and increased DNA degradation in ALS spinal cord motor neurons. Acta Neurol. Scand. 100:317-321.

41. Migheli, A., Cavalla, P., Marino, S., and Schiffer, D. 1994. A study of apoptosis in normal and pathologic nervous tissue after in situ endlabeling of DNA strand breaks. J. Neuropathol. Exp. Neurol. 53:606-616. 
42. He, B.P., and Strong, M.J. 2000. Motor neuronal death in sporadic amyotrophic lateral sclerosis (ALS) is not apoptotic. A comparative study of ALS and chronic aluminium chloride neurotoxicity in New Zealand white rabbits. Neuropathol. Appl. Neurobiol. 26:150-160.

43. Hiraishi, K., Suzuki, K., Hakomori, S., and Adachi, M. 1993. Le(y) antigen expression is correlated with apoptosis (programmed cell death). Glycobiology. 3:381-390.

44. Suurmeijer, A.J., van der Wijk, J., van Veldhuisen, D.J., Yang, F., and Cole, G.M. 1999. Fractin immunostaining for the detection of apoptotic cells and apoptotic bodies in formalin-fixed and paraffin-embedded tissue. Lab. Invest. 79:619-620.

45. Rangnekar, V.M. 1998. Apoptosis mediated by a novel leucine zipper protein Par-4. Apoptosis. 3:61-66.

46. Pedersen, W.A., Luo, H., Kruman, I., Kasarskis, E., and Mattson, M.P. 2000. The prostate apoptosis response-4 protein participates in motor neuron degeneration in amyotrophic lateral sclerosis. FASEB $J$. 14:913-924.

47. Harper, S.J., and LoGrasso, P. 2001. Signaling for survival and death in neurones. The role of stress-activated kinases, JNK and p38. Cell. Signal. 13:299-310.

48. Gupta, S. 2001. Molecular steps of death receptor and mitochondrial pathways of apoptosis. Life Sci. 69:2957-2964

49. Raoul, C., et al. 2002. Motoneuron death triggered by a specific pathway downstream of Fas. Potentiation by ALS-Linked SOD1 mutations. Neuron. 35:1067-1083.

50. Higgins, C.M., Jung, C., Ding, H., and Xu, Z. 2002. Mutant Cu, Zn superoxide dismutase that causes motoneuron degeneration is present in mitochondria in the CNS. J. Neurosci. 22:RC215.

51. Kong, J.M., and Xu, Z.S. 1998. Massive mitochondrial degeneration in motor neurons triggers the onset of amyotrophic lateral sclerosis in mice expressing a mutant SOD1. J. Neurosci. 18:3241-3250.

52. Browne, S.E., et al. 1998. Metabolic dysfunction in familial, but not sporadic, amyotrophic lateral sclerosis. J. Neurochem. 71:281-287.

53. Kroemer, G., and Reed, J.C. 2000. Mitochondrial control of cell death. Nat. Med. 6:513-519.

54. Warita, H., et al. 2001. Early decrease of survival signal-related proteins in spinal motor neurons of presymptomatic transgenic mice with a mutant SOD1 gene. Apoptosis. 6:345-352.

55. Alexianu, M.E., Kozovska, M., and Appel, S.H. 2001. Immune reactivity in a mouse model of familial ALS correlates with disease progression. Neurology. 57:1282-1289.

56. Nguyen, M.D., Julien, J.P., and Rivest, S. 2001. Induction of proinflammatory molecules in mice with amyotrophic lateral sclerosis: no requirement for proapoptotic interleukin-1beta in neurodegeneration. Ann. Neurol. 50:630-639.

57. Li, M., et al. 2000. Functional role of caspase- 1 and caspase- 3 in an ALS transgenic mouse model. Science. 288:335-339.

58. Chao, D.T., and Korsmeyer, S.J. 1998. BCL-2 family: regulators of cell death. Annu. Rev. Immunol. 16:395-419.

59. Mu, X., He, J., Anderson, D.W., Trojanowski, J.Q., and Springer, J.E. 1996.
Altered expression of bcl-2 and bax mRNA in amyotrophic lateral sclerosis spinal cord motor neurons. Ann. Neurol. 40:379-386.

60. Vukosavic, S., Dubois-Dauphin, M., Romero, N., and Przedborski, S. 1999. Bax and Bcl-2 interaction in a transgenic mouse model of familial amyotrophic lateral sclerosis. J. Neurochem. 73:2460-2468.

61. Troost, D., Aten, J., Morsink, F., and De Jong, J.M.B.V. 1995. Apoptosis in amyotrophic lateral sclerosis is not restricted to motor neurons. Bcl-2 expression is increased in unaffected post-central gyrus. Neuropathol. Appl. Neurobiol. 21:498-504.

62. Gonzalez de Aguilar, J.L., et al. 2000. Alteration of the Bcl-x/Bax ratio in a transgenic mouse model of amyotrophic lateral sclerosis: evidence for the implication of the p53 signaling pathway. Neurobiol. Dis. 7:406-415.

63. Guégan, C., Vila, M., Rosoklija, G., Hays, A.P., and Przedborski, S. 2001. Recruitment of the mitochondrial-dependent apoptotic pathway in amyotrophic lateral sclerosis. J. Neurosci. 21:6569-6576.

64. Kostic, V., Jackson-Lewis, V., De Bilbao, F., Dubois-Dauphin, M., and Przedborski, S. 1997. Bcl-2: prolonging life in a transgenic mouse model of familial amyotrophic lateral sclerosis. Science. 277:559-562.

65. Guégan, C., et al. 2002. Instrumental activation of Bid by caspase- 1 in a transgenic mouse model of ALS. Mol. Cell. Neurosci. 20:553-562.

66. Shinoe, T., et al. 2001. Upregulation of the pro-apoptotic BH3-only peptide harakiri in spinal neurons of amyotrophic lateral sclerosis patients. Neurosci. Lett. 313:153-157.

67. Miyashita, T., et al. 1994. Tumor suppressor p53 is a regulator of bcl-2 and bax gene expression in vitro and in vivo. Oncogene. 9:1799-1805.

68. Appella, E., and Anderson, C.W. 2001. Post-translational modifications and activation of p53 by genotoxic stresses. Eur. J. Biochem. 268:2764-2772.

69. Martin, L.J. 2000. p53 is abnormally elevated and active in the CNS of patients with amyotrophic lateral sclerosis. Neurobiol. Dis. 7:613-622.

70. Prudlo, J., et al. 2000. Motor neuron cell death in a mouse model of FALS is not mediated by the p53 cell survival regulator. Brain Res. 879:183-187.

71. Kuntz, C., Kinoshita, Y., Beal, M.F., Donehower, L.A., and Morrison, R.S. 2000. Absence of p53: no effect in a transgenic mouse model of familial amyotrophic lateral sclerosis. Exp. Neurol. 165:184-190.

72. Pasinelli, P., Borchelt, D.R., Houseweart, M.K., Cleveland, D.W., and Brown, R.H.J. 1998. Caspase- 1 is activated in neural cells and tissue with amyotrophic lateral sclerosis-associated mutations in copper-zinc superoxide dismutase. Proc. Natl. Acad. Sci. USA. 95:15763-15768.

73. Friedlander, R.M., Brown, R.H., Gagliardini, V., Wang, J., and Yuan, J. 1997. Inhibition of ICE slows ALS in mice. Nature. 388:31.

74. Nakagawa, T., et al. 2000. Caspase-12 mediates endoplasmic-reticulumspecific apoptosis and cytotoxicity by amyloid-beta. Nature. 403:98-103.

75. Bergeron, L., et al. 1998. Defects in regulation of apoptosis in caspase-2deficient mice. Genes Dev. 12:1304-1314.

76. Zhu, S., et al. 2002. Minocycline inhibits cytochrome c release and delays progression of amyotrophic lateral sclerosis in mice. Nature. 417:74-78.

77. Zheng, T.S., et al. 2000. Deficiency in caspase-9 or caspase-3 induces compensatory caspase activation. Nat. Med. 6:1241-1247. 